\title{
Divergent methylation of CRISPR repeats and cas genes in a subtype I-D CRISPR-Cas- system
}

Ingeborg Scholz ${ }^{1}$, Steffen C. Lott ${ }^{1}$, Juliane Behler ${ }^{1}$, Katrin Gärtner ${ }^{2}$, Martin Hagemann ${ }^{2}$ and Wolfgang R. Hess ${ }^{1,3^{*}}$ (D)

\begin{abstract}
Background: The presence and activity of CRISPR-Cas defense systems is a hallmark of many prokaryotic microorganisms. Here, the distribution of sequences related to the highly iterated palindrome 1 (HIP1) element and the DNA methylation of CGATCG motifs embedded within HIP1 as a vital part of the CRISPR1 repeat sequence was analyzed in the cyanobacterium Synechocystis sp. PCC 6803. Previously suggested functions of HIP1 include organization of chromosomal structure, DNA recombination or gene regulation, all of which could be relevant in CRISPR-Cas functionality.
\end{abstract}

Results: The CRISPR1 repeat-spacer array contains more than 50 CGATCG elements that are double-methylated $\left.{ }^{5 \mathrm{~m}} \mathrm{CG}^{6 \mathrm{~m}} \mathrm{ATCG}\right)$ by the enzymes M.Ssp6803I and M.Ssp6803III. Hence, more than 200 possible methylation events cluster over a stretch of $3600 \mathrm{bp}$ of double-stranded DNA. Bisulfite sequencing showed that these motifs were highly methylated at the ${ }^{\mathrm{m} 5}$ CGATCG positions whereas specific motifs within the CRISPR1 cas genes were hypomethylated suggesting a lowered accessibility for the DNA methylase to these regions. Assays for conjugation and CRISPR1-mediated DNA interference revealed a 50\% drop in conjugation efficiency in the mutant lacking the ${ }^{5 \mathrm{~m}} \mathrm{C}$ methylation of CGATCG motifs, while the highly efficient DNA interference activity was not affected by the lack of ${ }^{m 5}$ CGATCG DNA-methylation, nor was the capability to differentiate between self and non-self targets based on the protospacer adjacent motifs (PAMs) GTA and GTC versus the non-PAM AGC. A third DNA methylation mediated by M.Ssp6803II modifies the first cytosine in the motif GGCC yielding $G G^{m 4} C C$. We found a remarkable absence of GGCC motifs and hence the corresponding methylation over an $11 \mathrm{~kb}$ stretch encompassing all the cas genes involved in interference and crRNA maturation but not adaptation of the CRISPR1 system.

Conclusions: The lack of GGCC tetranucleotides along the CRISPR1 interference and maturation genes supports the reported hybrid character of subtype I-D CRISPR-Cas systems. We report tight and very high ${ }^{5 \mathrm{~m}} \mathrm{C}$ methylation of the CRISPR1 repeat sequences. Nevertheless, cells lacking the ${ }^{5 \mathrm{~m} C}$ methylation activity were unaffected in their CRISPR1mediated interference response but the efficiency of conjugation was reduced by $50 \%$. These results point to an unknown role of ${ }^{m 5}$ CGATCG DNA-methylation marks in conjugation and DNA transformation.

Keywords: CRISPR-Cas, Cyanobacteria, DNA methyltransferase, Highly iterated palindrome 1, HIP1, DNA methylation

\footnotetext{
* Correspondence: wolfgang.hess@biologie.uni-freiburg.de

${ }^{1}$ Faculty of Biology, Genetics an Experimental Bioinformatics, University of

Freiburg, Schänzlestr. 1, D-79104 Freiburg, Germany

${ }^{3}$ University of Freiburg, Freiburg Institute for Advanced Studies, Albertstr. 19,

D-79104 Freiburg, Germany

Full list of author information is available at the end of the article
}

(c) The Author(s). 2019 Open Access This article is distributed under the terms of the Creative Commons Attribution 4.0 International License (http://creativecommons.org/licenses/by/4.0/), which permits unrestricted use, distribution, and reproduction in any medium, provided you give appropriate credit to the original author(s) and the source, provide a link to the Creative Commons license, and indicate if changes were made. The Creative Commons Public Domain Dedication waiver (http://creativecommons.org/publicdomain/zero/1.0/) applies to the data made available in this article, unless otherwise stated. 


\section{Background}

The highly iterated palindrome 1 (HIP1) element 5'GCGATCGC-3' is an octameric palindromic repeat that is overrepresented in several cyanobacteria $[1,2]$. In the chromosome of the cyanobacterial model Synechocystis sp. PCC 6803 (from here: Synechocystis 6803) HIP1 instances occur at the frequency of one copy in every $1131 \mathrm{bp}[1,3]$. Statistical analyses supported the hypothesis that HIP1 motifs are maintained by selection, suggesting that HIP1 motifs likely perform biological functions [4]. A relation between the presence of HIP1 motifs and DNA recombination and/or repair processes has been suggested [5]. In addition or alternatively, a potential HIP1 function associated with chromosomal structure or maintenance was suggested based on its distribution along the chromosome [4].

At its core, the HIP1 element contains the recognition sequence of Dam DNA methyltransferases. These N6adenine-specific enzymes modify the adenosine residue within the target sequence GATC and are often essential for viability [6]. Methylation at the position $\mathrm{G}^{\mathrm{m} 6} \mathrm{ATC}$ in Synechocystis 6803 is carried out by the DNA methyltransferase M.Ssp6803III encoded by gene slr1803, which was found to be essential for viability of this cyanobacterium [7]. Moreover, the first cytosine within the HIP1 sequence is ${ }^{\mathrm{m} 5} \mathrm{C}$-methylated in Synechocystis 6803 by the DNA methyltransferase M.Ssp6803I encoded by slr0214 [7, 8]. Hence, in this cyanobacterium, the hexanucleotide $5^{\prime}$ CGATCG-3' within the HIP1 element can be methylated at four individual positions on the two DNA strands. Similar methylation patterns of HIP1 sequences have been reported for Anabaena sp. PCC 7120 [9]. In addition, the DNA methyltransferase M.Ssp6803II, encoded by sll0729, was recognized to methylate the first cytosine in the frequently occurring motif GGCC at the ${ }^{\mathrm{m} 4} \mathrm{C}$ position yielding $\mathrm{GG}^{\mathrm{m} 4} \mathrm{CC}$ [7]. GGCC is the most frequent methylation motif in Synechocystis 6803, on average providing one methylation site every $185 \mathrm{bp}$ on the chromosome.

Clustered regularly interspaced short palindromic repeats (CRISPRs)-Cas systems are adaptive immune systems in bacteria and archaea that use CRISPR RNAs (crRNAs) as guides and CRISPR-associated proteins (Cas) for antiviral defense [10-13]. There are three different CRISPR-Cas systems in Synechocystis 6803 [14]. Based on the associated cas gene complement, these systems were classified as one subtype I-D (CRISPR1), one subtype III-D (CRISPR2) and one subtype III-Bv (CRISPR3) CRISPR-Cas system [14, 15].

The crRNAs originate from the CRISPR repeatspacer arrays initially in the form of long precursor transcripts. After transcription, the CRISPR repeats are recognized by processing maturases. These frequently belong to the Cas6 class of endoribonucleases [16] whereas in subtype I-C systems the endoribonuclease is Cas5d [17, 18]. In case of Synechocystis 6803, crRNA maturation proceeds by the Cas6-1 enzyme for the CRISPR1 system and by Cas6-2a for the CRISPR2 system [14, 19, 20], while for the CRISPR3 system RNase E was recognized as the major maturation endoribonuclease [15]. During interference, crRNAs guide the proteinaceous CRISPR effector complex to their targets, also known as protospacers, resulting in efficient immunity against potentially harmful invading nucleic acids [21-23]. CRISPR1 interference activity was shown to strictly depend on the presence of a DNA sequence element called protospacer adjacent motif (PAM). PAM sequences are functionally critical for CRISPR-based immune systems and are located adjacent to each protospacer consisting of a short signature sequence of 2-5 nt, depending on the CRISPR type and organism. The PAM sequences GTN were found to efficiently mediate CRISPR1 interference in Synechocystis 6803, while there also exist PAMs, e.g. the sequence motif AGC, that do not license interference [24].

While unexpected connections between bacterial natural competence, ubiquitin signaling and DNA modification was reported for type VI-B CRISPR-Cas systems [25], a possible relationship between DNA methylation and CRISPR-Cas-mediated interference responses has not been studied thus far. We noticed the CGATCG sequence to be overrepresented within the CRISPR1 system of Synechocystis 6803, with possibly more than 200 methylation events over a stretch of only $3600 \mathrm{nt}$. The recent availability of bisulfite sequencing data for this organism [7] enabled the detailed analysis of pSYSA cytosine methylation. Therefore, here we investigated the DNA methylation of the CRISPR1 system within the context of the pSYSA plasmid and whether there was a correlation between DNA methylation and DNA interference in a conjugation-based assay using a DNA methyltransferase mutant.

\section{Methods}

\section{Strains and culture conditions}

Synechocystis sp. 6803 was maintained on BG11 mineral medium [26] at $30^{\circ} \mathrm{C}$ under constant illumination. The slr0214 deletion mutant $(\Delta s$ lr0214-A1) and the slr0214 insertion mutant $(\Delta$ slr0214-B1) were described previously [7, 8]. Liquid cultures of Synechocystis 6803 wild type and slr0214 mutants were grown photoautotrophically in volumes of $50 \mathrm{~mL}$ in Erlenmeyer flasks in BG11 medium, with shaking at $50 \mu \mathrm{mol}$ photons $\mathrm{s}^{-1} \mathrm{~m}^{-2}$ at $30^{\circ} \mathrm{C}$. E. coli cultures were grown in LB medium at $37^{\circ} \mathrm{C}$. Growth was followed by measurements of the optical density at $750 \mathrm{~nm}\left(\mathrm{OD}_{750}\right)$ for Synechocystis 6803 and at $600 \mathrm{~nm}\left(\mathrm{OD}_{600}\right)$ for E. coli. 


\section{Synechocystis 6803 conjugation and interference assay} Conjugation between E. coli and Synechocystis 6803 by triparental mating was essentially carried out as described previously [14]. In short, flasks containing LB medium without antibiotics were inoculated with overnight cultures of E. coli J53/RP4 (helper strain) and DH5 $\alpha$ with the plasmid of interest (donor strain) to obtain an $\mathrm{OD}_{600}$ of 0.1 and incubated at $37^{\circ} \mathrm{C}$ with shaking at $180 \mathrm{rpm}$ for $2.5 \mathrm{~h}$. Plasmid-bearing and helper strains equivalent to an $\mathrm{OD}_{600}$ of 7.0 were harvested by centrifugation, resuspended in $1 \mathrm{~mL} \mathrm{LB}$, combined $(1 \mathrm{~mL}$ plasmid-bearing and $1 \mathrm{~mL}$ helper strain), centrifuged again, resuspended in $100 \mu \mathrm{L} \mathrm{LB}$ and incubated at $30^{\circ} \mathrm{C}$ without shaking for $1 \mathrm{~h}$. In parallel, cultures of the Synechocystis 6803 wild type and slr0214 deletion strains [7] equivalent to an $\mathrm{OD}_{750}$ of 0.75 were harvested by centrifugation, resuspended in $800 \mu \mathrm{L}$ BG11 medium and combined with the E. coli cells. The resulting mix was centrifuged, resuspended in $30 \mu \mathrm{L}$ BG11 and placed on a sterile filter located on a BG11 agar plate supplemented with $5 \% \mathrm{LB}$ medium at $30^{\circ} \mathrm{C}$ overnight (slightly covered with tissue). The next morning the filter was rinsed with $400 \mu \mathrm{L}$ BG11 medium, $20 \mu \mathrm{L}$ and $40 \mu \mathrm{L}$ of the cell suspension were plated, respectively, on BG11 plates containing $5 \mu \mathrm{g} \mathrm{mL}{ }^{-1}$ gentamicin.

Interference assays essentially were performed as described $[15,24]$ using tri-parental mating with the selfreplicating conjugative vector pVZ322 [27] containing the gentamicin resistance cassette for selection plus a protospacer sequence to spacer 1 of the CRISPR 1 system with either of the PAMs GTA, GTC or the non-PAM AGC as a control. These plasmids were constructed during previous work [24].

The Synechocystis 6803 wild type, the slr0214 deletion strain ( $\Delta$ slr0214-A1) and the slr0214 insertion strain ( $\Delta$ slr0214-B1) [7] were used as recipients. Transconjugant colonies were counted after incubation at $30^{\circ} \mathrm{C}$ for 2 weeks. Experiments were performed in biological triplicates for the plasmid targets (pT) and in parallel with the control plasmids ( $\mathrm{pNT}$ ) either without protospacer sequence or containing the AGC non-PAM fused to a protospacer.

\section{Methylation analysis by bisulfite sequencing}

The bisulfite sequencing data were obtained in the frame of a previous study [7]. In short, $200 \mathrm{ng}$ of DNA were bisulfite treated with the Zymo Gold kit (Zymo Research, Cat. No. D5005) and libraries constructed using the Ovation Ultra-Low Methyl-Seq library kit (NuGEN Cat. No. 0535-32) according to the manufacturer's instructions, followed by sequencing on the Illumina HiSeq2500 system yielding 2,559,017 raw reads. The sequences were-quality filtered and adaptertrimmed using Trimmomatic v0.36 [28] and FastQC v0.67 (http://www.bioinformatics.babraham.ac.uk/projects/fastqc/) leaving 2,552,913 reads for further analysis. For mapping to the Synechocystis 6803 pSYSA plasmid and quantitative evaluation, Bismark v0.17 was used with default options [29] in conjunction with Bowtie 2 [30]. The raw sequencing data can be accessed at https:// www.ncbi.nlm.nih.gov/biosample/8378604 (BioProject ID: PRJNA430784, BioSample: SAMN08378604, Run: SRX3574087).

\section{DNA manipulation and hybridization}

For total DNA extraction from the Synechocystis 6803 wild type, $\Delta$ slr0214-A1 and $\Delta$ slr0214-B1 strains, cultures of $50 \mathrm{~mL}$ were harvested by centrifugation for $5 \mathrm{~min}$. The pellet was resuspended in $1 \mathrm{~mL}$ SET buffer $(50 \mathrm{mM}$ Tris, pH 7.5; $1 \mathrm{mM}$ EDTA, pH 8; 25\% (w/v) sucrose) and shock frozen in liquid nitrogen. For lysis, cells were incubated overnight at $50^{\circ} \mathrm{C}$ in $100 \mathrm{mM}$ EDTA (pH 8), $2 \%$ SDS (w/v) and $100 \mu \mathrm{g} / \mathrm{mL}$ proteinase K. After lysis, DNA was extracted twice by adding 1 vol phenol/ chloroform/isoamylalcohol (25:24:1) and once by adding 1 vol chloroform/isoamylalcohol (24:1). After each addition, the solution was mixed and phase separation was achieved by centrifugation ( $6 \mathrm{~min}, 6000 \mathrm{rpm}$ ) in a swing-out rotor. The upper aqueous phase was removed and DNA was precipitated by addition of 1 vol isopropanol and incubation at $-20^{\circ} \mathrm{C}$ overnight. DNA was collected by centrifugation $(13,000 \mathrm{rpm})$ for $30 \mathrm{~min}$ at $4{ }^{\circ} \mathrm{C}$. The pellet was washed with $70 \%$ ethanol, air-dried and resuspended in $50 \mu \mathrm{L}$ sterile Milli-Q water. DNA concentration was measured in a NanoDrop spectrophotometer (ND-1000, peQLab). The quality and quantity of nucleic acid extraction was verified optically in ethidium bromide stained $0.8 \%$ agarose gels.

For restriction analysis, $10 \mu \mathrm{g}$ each of total DNA from Synechocystis 6803, $\Delta$ slr0214-A1 and $\Delta s l r 0214-\mathrm{B} 1$ were digested with $P v u \mathrm{I}, D p n \mathrm{I}$ or Sau3AI. Restriction endonucleases were used in a 10-fold excess and incubated overnight at $37^{\circ} \mathrm{C}$ to ensure complete digestion. Heat inactivation was performed at $80^{\circ} \mathrm{C}(P v u \mathrm{I}$ and $D p n \mathrm{I})$ and $65^{\circ} \mathrm{C}$ (Sau3AI) for $20 \mathrm{~min}$. Digested total DNA was separated overnight on an ethidium bromide stained $1.2 \%$ agarose gel under a field strength of $1.8 \mathrm{~V} \mathrm{~cm}^{-1}$ at $4{ }^{\circ} \mathrm{C}$. The gel was incubated in denaturation solution $(1.5 \mathrm{M}$ $\mathrm{NaCl}, 0.5 \mathrm{M} \mathrm{NaOH}$ ) at $70 \mathrm{rpm}$ at room temperature for $30 \mathrm{~min}$ and subsequently in neutralization solution (1.5 $\mathrm{M} \mathrm{NaCl}, 0.5 \mathrm{M}$ Tris $\mathrm{pH} 7.5$ ) for the same time. DNA was transferred to Hybond- $\mathrm{N}+$ membranes (Amersham Cat. No. RPN303B) with 10x saline sodium citrate (3 M $\mathrm{NaCl}, 300 \mathrm{mM}$ sodium citrate $\mathrm{pH}$ 7) transfer buffer by capillary blotting overnight. After blotting the DNA was crosslinked to the membrane with $125 \mathrm{~mJ}$ using a UVStratalinker (Stratagene). 
The following synthetic primers were used to generate the DNA probe for Southern hybridization by PCR: SS slr7010_fw, 5`-CCAAGAACGTCAGCAAACCCAAAC3' and SS_slr7010_rev, 5'-CCATCCCAAATCCCTGACTGTAAAG-3'. PCR amplification was performed with Q5 high fidelity DNA polymerase (NEB Cat. No.

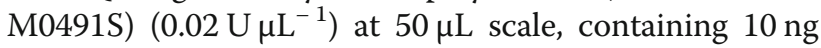
of template DNA, $200 \mu \mathrm{M}$ dNTPs, $0.5 \mu \mathrm{M}$ of each primer and $10 \mu \mathrm{L}$ of $5 \mathrm{x}$ Q5 reaction buffer. The initial denaturation temperature was $98^{\circ} \mathrm{C}$ for $30 \mathrm{~s}$. Thirty-two cycles were performed at $98^{\circ} \mathrm{C}$ for $10 \mathrm{~s}, 60^{\circ} \mathrm{C}$ for $30 \mathrm{~s}$ and $72{ }^{\circ} \mathrm{C}$ for $18 \mathrm{~s}$, followed by a 2 min final extension at $72^{\circ} \mathrm{C}$. The PCR product was loaded on a Midori green (Nippon Genetics Cat. No. MG04) stained 1.8\% agarose gel and the correct band was gel-eluted using the gel extraction kit from Macherey-Nagel (Cat. No. 740609.10).

For Southern hybridization, $25 \mathrm{ng}$ of DNA probe was labeled with $\alpha^{32} \mathrm{P}$-dCTP using the Random Primers DNA Labeling System from Thermo Fisher Scientific (Cat. No. 18187013). Hybridization was performed overnight at $58{ }^{\circ} \mathrm{C}$ in Southern hybridization buffer (250 $\mathrm{mM}$ NaPi-buffer $\mathrm{pH}=7.2,7 \%$ SDS, $250 \mathrm{mM} \mathrm{NaCl}$ ) followed by $10 \mathrm{~min}$ wash steps each in wash buffers 1 (2x SSC, $1 \%$ SDS), 2 ( $1 x$ SSC, $0.1 \%$ SDS) and $3(0.1 x$ $\mathrm{SSC}, 0.1 \% \mathrm{SDS}$ ) at $53^{\circ} \mathrm{C}$. The signals were detected with a storage phosphor screen (Kodak) and a GE Healthcare Typhoon FLA 9500 imaging system.

\section{Results}

\section{Distribution and methylation of GGCC motifs on the} pSYSA plasmid

We used the bisulfite sequencing data that were obtained in the frame of the global characterization of DNA methyltransferases in Synechocystis 6803 [7] to evaluate the degree of $\mathrm{GG}^{4 \mathrm{~m}} \mathrm{CC}$ methylation on plasmid pSYSA (Fig. 1). Bisulfite sequencing permits the direct and highly sensitive detection of 5-methylcytosines, but it can also be used to map 4-methylcytosines; although ${ }^{\mathrm{m} 4} \mathrm{C}$ is partially resistant to bisulfite-mediated deamination and therefore the assay is less sensitive [31]. There

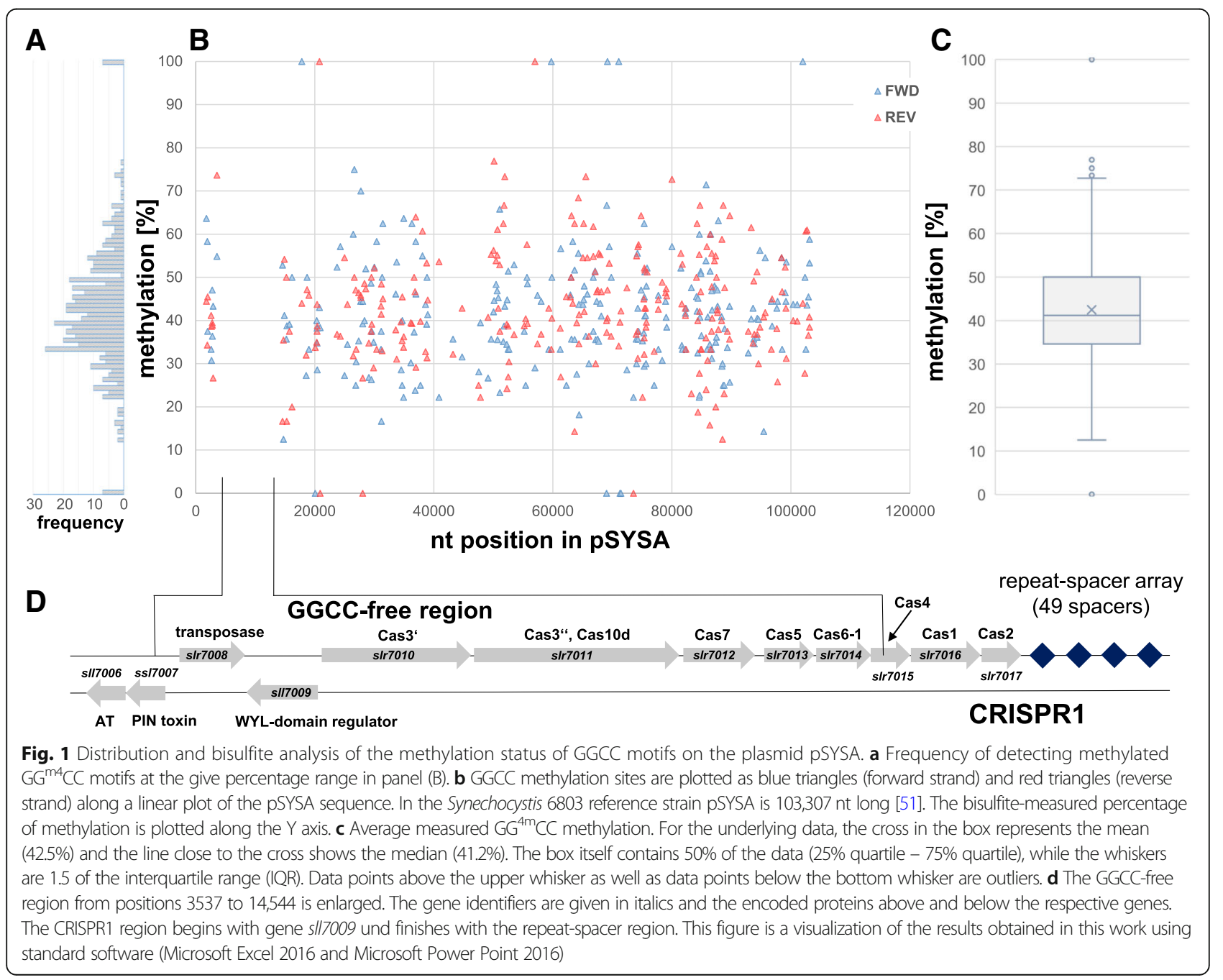


are 246 GGCC motifs on plasmid pSYSA per DNA strand, one every $418 \mathrm{nt}$. The measured $\mathrm{GG}^{4 \mathrm{~m}} \mathrm{CC}$ methylation was $42 \%$ on average (Fig. 1c). This value matches the reported average sensitivity for the detection of this DNA modification by bisulfite sequencing [31]. The specificity of the detected $\mathrm{GG}^{4 \mathrm{~m}} \mathrm{CC}$ methylation was verified by analyzing a sll0729 deletion mutant lacking M.Ssp6803II activity in parallel, where we found zero methylation at these sites (not shown). The GGCC sites are relatively randomly distributed on pSYSA with one important exception, a region entirely free of this motif ranging from position 3537 to position 14,544 (Fig. 1b). The GGCC-free region starts in the gene ssl7007 encoding the toxin component of a toxin-antitoxin system [32] and finishes within slr7015 (cas4) (Fig. 1d). Hence, this stretch encompasses all the genes belonging to the iCas and pCas functional modules according to the classification suggested by Roger Garrett [33] and separating them from the cas4-1-2 cassette making up the aCas module for adaptation. The GGCC-free sequence also contains slr7008 encoding an IS4 transposase and sll7009 encoding a transcriptional repressor [34] that possesses a WYL domain [35].

\section{High methylation of CRISPR1 repeats and}

\section{hypomethylation of cas genes at CGATCG sites}

We detected one CGATCG sequence that is recognized by the DNA methyltransferases M.Ssp6803I and M.Ssp6803III (recognizing the internal GATC [7]) within every single of the CRISPR1 repeats of Synechocystis 6803. All motif instances are preceded by a $G$ residue, hence matching seven of the eight nucleotides of the HIP element (GCGATCGC) (Fig. 2). After transcription, the
CRISPR repeats are recognized by processing maturases, which frequently belong to the Cas6 class of endoribonucleases [16]. This maturation step is obligatory for successful interference and often involves recognition of a repeat-internal stem-loop secondary structure [36]. In case of the Synechocystis 6803 CRISPR1 system this step is performed by the Cas6-1 enzyme [14, 20]. Therefore, we judged the location of the repeated CGATCG sequences with regard to the repeat secondary structure after transcription. The repeat-internal CGATCG motifs after transcription form 4 of the 5 unpaired loop nucleotides and two nucleotides of the right arm of the RNA stem (Fig. 2). Hence, this motif is part of the sequence that has been found to be functionally relevant for subsequent recognition by the Cas6-1 maturation endonuclease.

The CRISPR1 system of Synechocystis 6803 consists of 50 repeats and 49 spacers in the reference strain [14]. The motif CGATCG contains two methylated bases, the first cytosine methylated by M.Ssp6803I and the adenosine methylated by M.Ssp6803III, together yielding ${ }^{5 \mathrm{~m}} \mathrm{CG}^{6 \mathrm{~m}}$ ATCG. Because the motif is palindromic, there are four possible methylation events per CGATCG within the DNA double strand. Hence, over a stretch of only $3600 \mathrm{nt}$, there are 200 possible methylation events for the DNA double strand due to the CRISPR1 repeats alone. Moreover, the CRISPR1 repeat-spacer array contains within its spacer sequences four GGCC and eight GATC sites, which are not part of the CGATCG motif, possibly containing additional methylated bases. This very tight clustering of DNA methyltransferase recognition sites might lead to an undermethylation when the enzyme was locally limiting, but this was not observed. On average, the methylation level of ${ }^{5 \mathrm{~m}}$ CGATCG
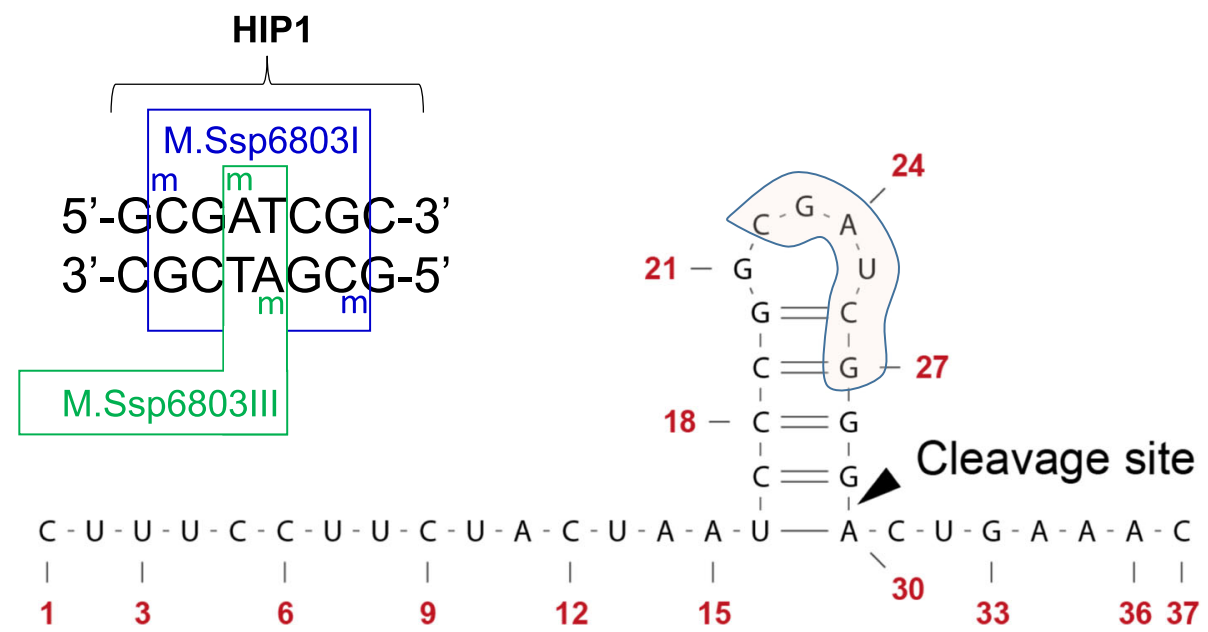

Fig. 2 Four DNA methylation events can occur within the 5'- CGATCG - 3' sequence that is at the core of the octameric HIP1 element. These methylations are carried out by the enzymes M.Ssp6803I (blue) and M.Ssp6803III (green) [7]. A 5'- CGATCG - 3' recognition sequence is located within every single repeat of the Synechocystis 6803 CRISPR1 system. After transcription, this recognition sequence corresponds to four of the five nucleotides within the crRNA loop and to the two upper nucleotides of the right arm within the repeat stem-loop secondary structure that is essential for recognition by the Cas6-1 endoribonuclease, cleaving at the indicated site [20] 
measured by bisulfite sequencing for the entire pSYSA forward strand was 90.5 and $88.6 \%$ for the reverse strand and it was especially high in the repeat-spacer region (Fig. 3). We used two different strains for this analysis, the wild type and a $\Delta$ sllo729 mutant that lacks M.Ssp6803II activity but not affecting ${ }^{5 \mathrm{~m}}$ CGATCG methylation. The measured methylation level of ${ }^{5 m}$ CGATCG in the repeat-spacer region reached 97.6 and $96 \%$ for the forward strand and 92.5 and $93 \%$ for the reverse strand each in the wild type and $\Delta$ sllo729 mutant, respectively $(n=50)$. We conclude that the level of ${ }^{5 \mathrm{~m}}$ CGATCG methylation in this region was close to saturation on the forward strand and on the reverse strand with the exception of repeats 22 and 44 as well (Fig. 3). The plasmid pSYSA exists in multiple copies, likely similar to the chromosome for which $\sim 20$ copies per cell were reported during exponential growth phase [37]. Therefore, there was a high chance of freshly replicated plasmid copies explaining the average methylation below $100 \%$.

However, we noticed striking imbalances of ${ }^{5 m}$ CGATCG methylation levels with regard to the CRISPR1 cas genes. The region encompassing all cas genes (from nt position 5000 to 16,100) showed hypomethylation of this motif with only 63.4 and $66.3 \%$ methylation for the forward and reverse strand). Methylation was nearly absent at the motif positions $7392 / 7397$ and 7998/8003 (Figs. 3 and 4). These positions reside within the slr7010 gene encoding the Cas3' protein (Fig. 4).

\section{Verification of CGATCG hypomethylation by southern hybridization}

The ${ }^{\mathrm{m} 5} \mathrm{C}$ hypomethylation at the CGATCG sites detected for the CRISPR1 cas genes by bisulfite sequencing was independently tested by Southern hybridization. Total DNA was isolated from the wild type, the slr0214 deletion mutant ( $\triangle$ slr0214-A1) and the slr0214 insertion mutant ( $\Delta$ slr0214-B1). For restriction analysis, $P v u \mathrm{I}$ was chosen that cuts CGATCG but is sensitive to the methylated ${ }^{\mathrm{m}}{ }^{5}$ CGATCG site. Hence, $P v u \mathrm{I}$ can only cleave when the cytosine in CGATCG is unmethylated, but it is not affected by methylation of the internal adenosine. For analysis, we chose the slr7010 gene encoding the Cas3' protein (Fig. 1). According to bisulfite analysis, CGATCG at positions $7392 / 7397$ and $7998 / 8003$ were nearly not ${ }^{\mathrm{m} 5} \mathrm{C}$ methylated, while the interspersed CGATCG at position 7839/7844 was methylated. Treatment by $P v u \mathrm{I}$ yielded two fragments, $447 \mathrm{bp}$ and $159 \mathrm{bp}$ in size with DNA from the two slr0214 mutants (Fig. 4), confirming the unmethylated status of all relevant sites. With DNA from the wild-type strain, these products were not obtained but a signal at $\sim 600 \mathrm{bp}$, which corresponds to the $606 \mathrm{bp}$ fragment consisting of both parts. Hence, this result validates the bisulfite analysis with mainly cytosineunmethylated CGATCG sites at positions 7392/7397 and

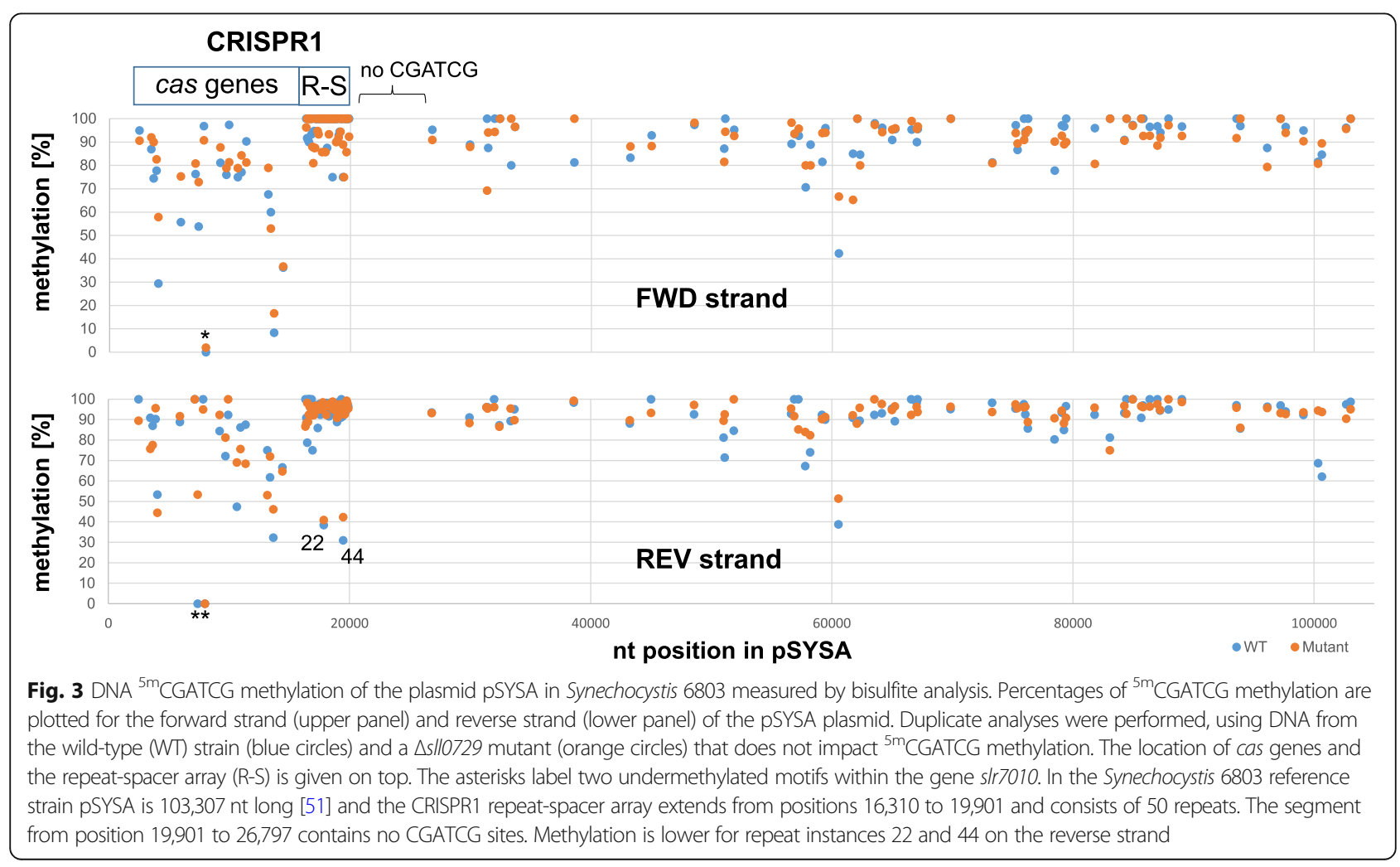



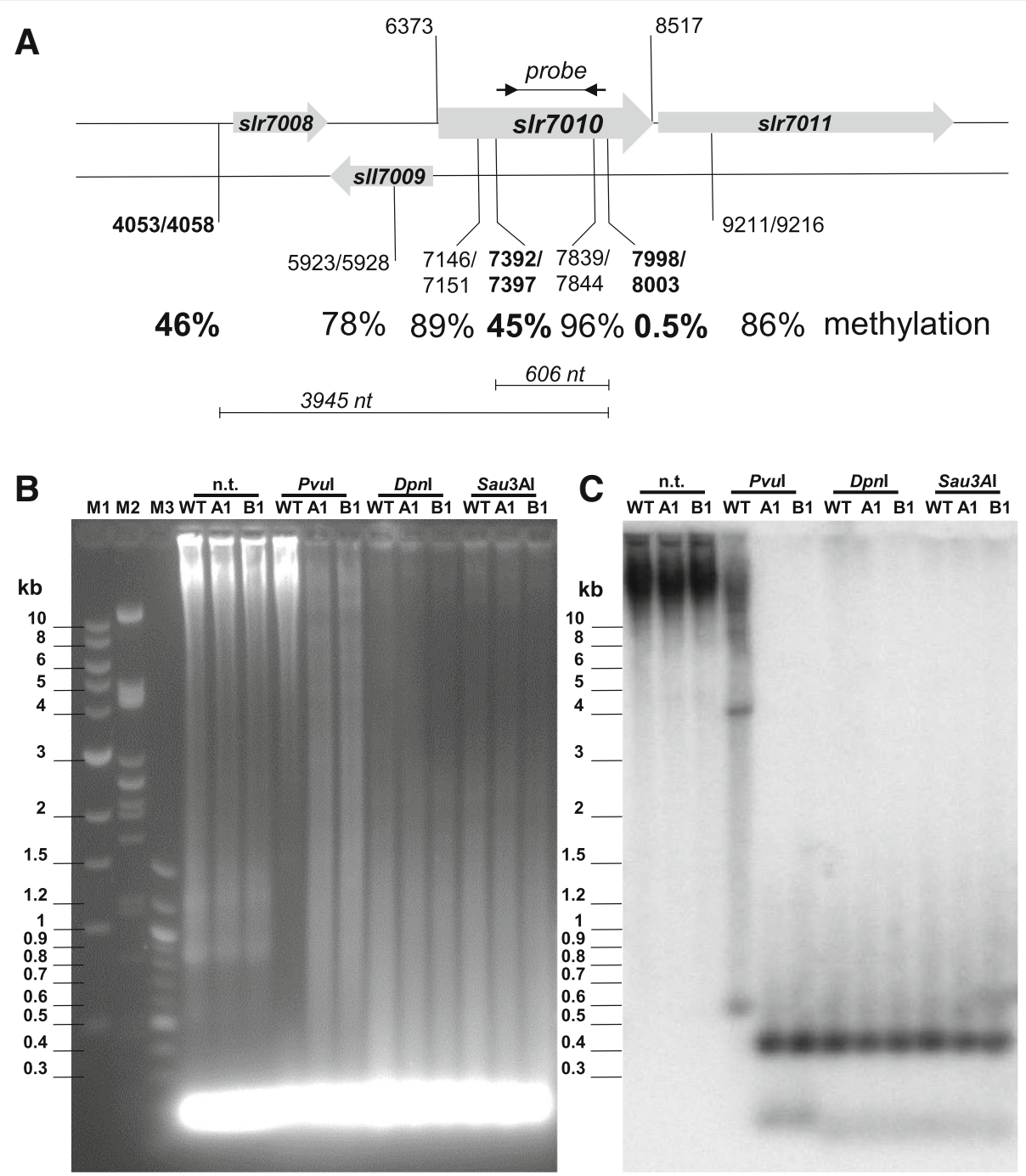

$\frac{\text { n.t. }}{\text { WT A1 B1 }} \frac{\text { Pvul }}{\text { WT A1 B1 }} \frac{\text { Dpnl }}{\text { WT A1 B1 }} \frac{\text { Sau3Al }}{\text { WT A1 B1 }}$

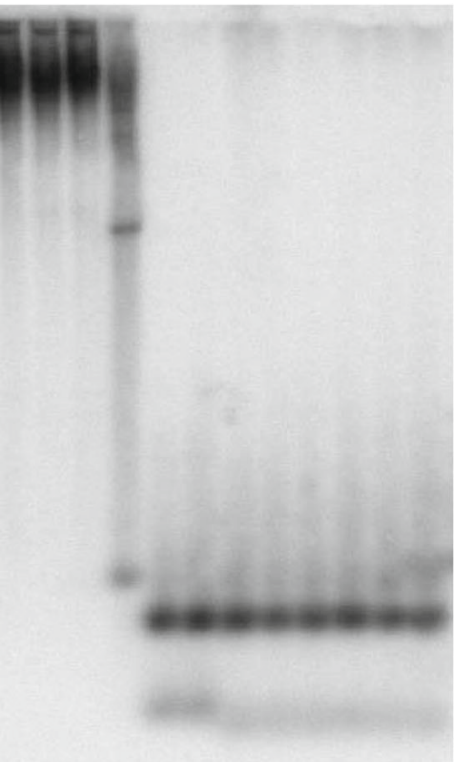

Fig. 4 Validation of ${ }^{5 m}$ CGATCG methylation status by Southern blot hybridization. a Scheme of the probed region from the CRISPR1 system of Synechocystis 6803 plasmid pSYSA and percentage of methylated cytosine residues at the indicated positions (first number, position on forward strand; second number, position on the reverse strand) according to bisulfite analysis. Undermethylated sites are bold-faced. The location of primers to generate the 600 bp probe for hybridization to s/r7010 DNA fragments is indicated by arrows. b Gel image of the DNA from wild type (WT) and the two s/r0214 mutants (A1 and B1) with no treatment (n.t.) and after restriction by Pvul, Dpnl or Sau3Al and separation by agarose gel electrophoresis. Three different markers were used as size standards, a $1 \mathrm{~kb}(\mathrm{M} 1)$ and a $100 \mathrm{nt}$-ladder (M3) and DNA of bacteriophage $\lambda$ after restriction by Pstl (M2). c Image of the blot resulting from the gel in panel (b) after Southern transfer and hybridization to the probe indicated in panel (a). The lengths of the two additional bands in Pvul-digested DNA from the wild type correspond to the predicted lengths indicated in panel (A) for the products of partial digestion between the sites at positions 4053/4058 or 7392/7397 and 7998/8003 due to the different methylation levels

7998/8003, while the interspersed CGATCG at position $7839 / 7844$ was methylated. In addition, a band at $\sim 3.9 \mathrm{~kb}$ was detected, which corresponds to another restriction fragment from position 4053/4058 to 7998/8003 (Fig. 4a). According to bisulfite sequencing, the cytosines at 4053/ 4058 were methylated at $46 \pm 12.7 \%(n=4)$. For control, aliquots of the same DNA samples were digested by $D p n \mathrm{I}$ and Sau3AI. DpnI cleaves only when its recognition site is Dam-methylated, G ${ }^{\mathrm{m} 6}$ ATC, whereas Sau3AI is insensitive to any of these methylations. The resulting restriction fragments were of identical lengths in all three samples, pointing to full $\mathrm{G}^{\mathrm{m} 6} \mathrm{ATC}$ methylation. The smaller fragment was somewhat shorter than the $159 \mathrm{bp}$ fragment generated by $P v u \mathrm{I}$, which is due to an internal GATC site, leading to a $30 \mathrm{bp}$ shorter fragment.

\section{Missing ${ }^{\text {m5 }}$ CGATCG methylation affects conjugation rates but not CRISPR1-mediated DNA interference}

Within this study, we identified specific imbalances in the distribution and methylation level of GGCC and 
CGATCG sites within the CRISPR1 system of Synechocystis 6803 . The tightly clustered appearance of many methylated bases within the repeat-spacer array could affect DNA interference. Therefore, we performed assays for conjugation and interference efficiency in wild type and the two different slr0214 mutant lines that lack the methylation activity of M.Ssp6803I encoded by this gene.

Due to the lack of identified bacteriophages infecting Synechocystis 6803, we used a conjugation-based assay. To trigger interference, we inserted a protospacer into the conjugative vector matching spacer 1 of the CRISPR1 system. In addition, we added different PAM sequences that should facilitate the differentiation between "self" and "non-self" targets [24]. The CRISPR1mediated DNA interference was as efficiently in the two different $\Delta$ slr0214 mutants as in the wild type control and no difference was observed regarding the different PAMs (Fig. 5a-c). In all combinations, the GTC and GTA PAMs facilitated DNA interference, while the AGC non-PAM did not and served as a control. We conclude that the absence of CRISPR $1{ }^{\mathrm{m} 5} \mathrm{C}$ methylation had no effect on the efficiency of DNA interference. However, a difference was noticed in the conjugation efficiencies, which were in all instances below 50\% compared to the wild type (Fig. 5d).

\section{Discussion}

In this work, we focused on the differential methylation on the plasmid pSYSA, but DNA methyltransferases can target any of their recognition sequences on the chromosome as well as on the other six plasmids. Synechocystis 6803 encodes five DNA methyltransferases. Two of these are encoded on plasmid pSYSX (M.Ssp6803IV and M.Ssp6803V) and three on the chromosome [7]. Among these DNA methyltransferases, M.Ssp6803I executes ${ }^{\mathrm{m} 5}$ CGATCG methylation, M.Ssp6803II performs $\mathrm{GG}^{4 \mathrm{~m}} \mathrm{CC}$ and M.Ssp6803III the dam-like $\mathrm{G}^{\mathrm{m} 6} \mathrm{ATC}$ methylation [7]. Missing cytosine N4-methylation of GGCC motifs in the $\Delta$ sll0729 mutant that lacks M.Ssp6803II activity resulted in strong phenotypical alterations, which were associated with the regulation of transcription, DNA replication and

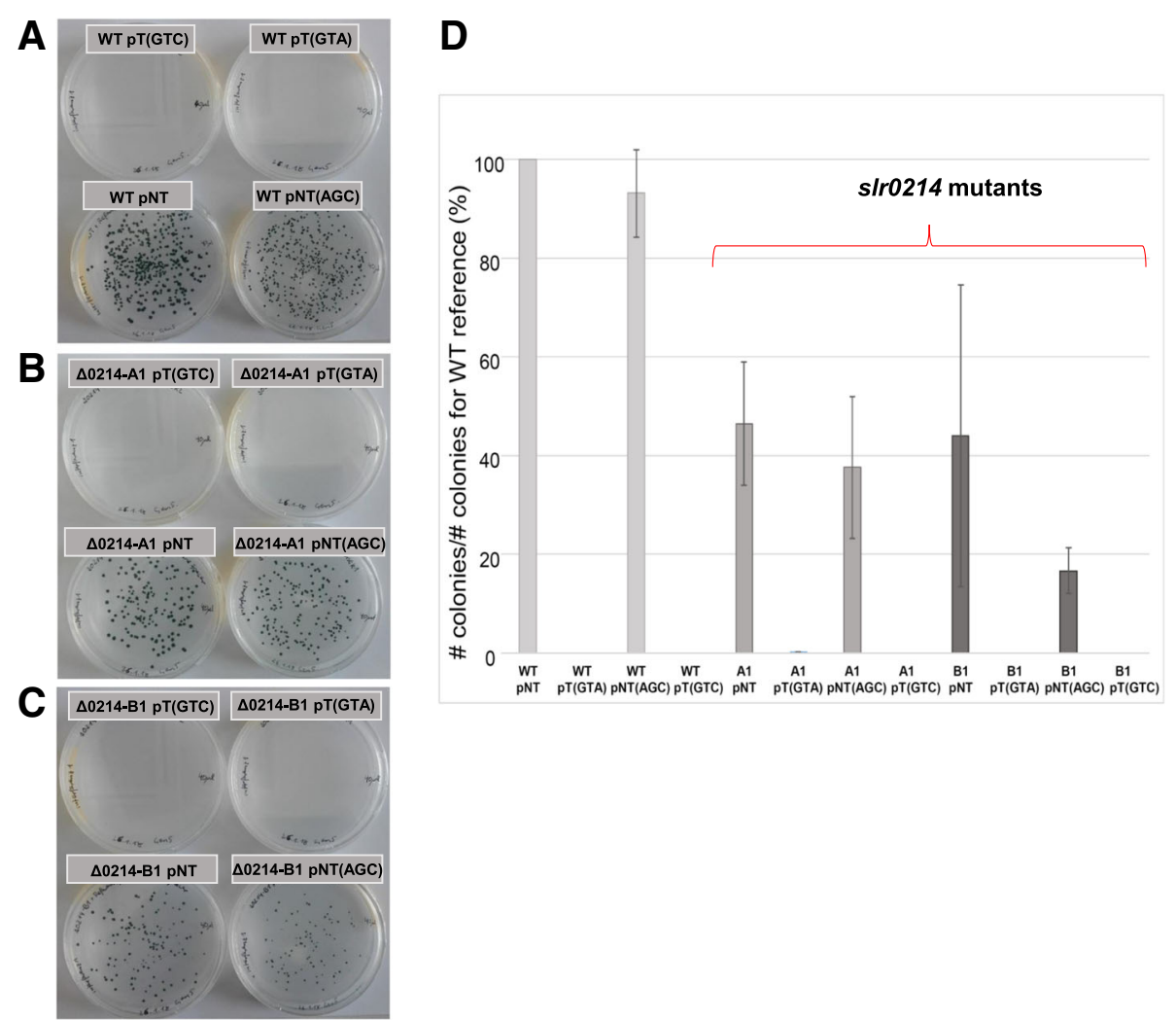

Fig. 5 Assays for conjugation and interference efficiency in wild type (WT) and slr0214 mutant cell lines. The conjugation efficiency was tested for plasmids carnying a protospacer to spacer 1 and a functional protospacer adjacent motif (PAM) of the CRISPR1 system and therefore were potential targets (labelled pT) or that lacked the protospacer or maintained a non-PAM fused to the protospacer and served as non-target controls (labelled pNT). The protospacer in the pT plasmids was linked to a functional GTC or GTA PAM that facilitates recognition of the invader DNA or to an AGC non-PAM that does not license interference in Synechocystis 6803 [24]. a Plates showing interference and growth of colonies following conjugation into wild type cells. b Assay in the $\Delta$ slro214-A1 deletion mutant. $\mathbf{c}$ Assay in the $\Delta$ slr0214-B1 insertion mutant. $\mathbf{d}$ Conjugation efficiencies (numbers of colonies normalized to the wild type with the pNT plasmid control). The bars represent the mean \pm SE of three biological replicates each 
DNA repair [38]. For the methylation of the HIP1-related motif via M.Ssp6803I, a role in DNA repair processes was previously suggested [5]. With regard to transformation, it was reported that the methylation of plasmid DNA in $E$. coli expressing M.Ssp6803I encoded by the Synechocystis 6803 gene slr0214 prior to transformation led to an 11- to 161-fold-higher efficiency in the subsequent integrative transformation of Synechocystis 6803 [39]. In contrast, expression of M.Ssp6803II from gene sll0729 methylating GGCC [7] had no measurable impact on transformation efficiencies [39]. Both of these DNA methyltransferases do not belong to a restriction-modification system. Therefore, the molecular basis for these observations has remained elusive.

During the study of methylation frequencies of single CGATCG sites, we made the remarkable observation that only a few sites were almost unmethylated, while the vast majority of sites was methylated almost $100 \%$. One virtually methyl-free CGATCG site is situated on the plasmid pSYSA within the CRISPR-Cas systems encoding genes, which raised our attention and initiated the detailed functional study reported here. CRISPR-Cas systems involve DNA recombination at their very heart. Degradation products of the RecBCD repair complex were found to serve as templates for spacer acquisition as new spacers [40], especially in the naïve spacer acquisition in E. coli [41], with the RecBCD helicase function as the most important activity [42]. However, also vice versa, cas1 gene deletions were found to affect chromosome segregation and to lead to increased sensitivity towards DNA damage [43]. Another, recently discovered, bacterial phage resistance system called bacteriophage exclusion (BREX) [44] distinguishes self from non-self DNA by methylation of a specific DNA site [45]. It should be noticed that the extended GCGATCGG motif present in CRISPR1 repeats constitutes a 1-nt deviation from the cognate HIP1 sequence but left the recognition sequence for the DNA methyltransferase M.Ssp6803III intact. For all these reasons, the connection between DNA methylation and the CRISPR-Cas apparatus is worth investigation.

We noticed a striking absence of GGCC sites among the iCas and pCas modules of the CRISPR1 system. The cas3,' cas $10 d$, cas7, cas5 and cas6-1 genes, together with the genes sll7009 and slr7008 for a WYL-domain regulator and a transposase, respectively, lack any GGCC site, which may indicate that they originate from another organism, possibly be transferred via horizontal gene transfer. Because this system belongs to the subtype I-D, this observation fits to the previously proposed hybrid character of this subtype, in which signature genes for a type I-C system were combined with a distinct type III gene arrangement [46].
Moreover, we show that the CRISPR1 system of Synechocystis 6803 contains M.Ssp6803III recognition sequences within its repeat sequences and that they are highly methylated while the associated cas genes showed hypomethylation at certain but not all ${ }^{\mathrm{m} 5}$ CGATCG sites. We verified the lowered methylation within the slr7010 gene encoding Cas3' for the positions $7392 / 7397$ and $7998 / 8003$ by Southern hybridization while the motif at position $7839 / 7844$ was methylated. The reduced or lacking DNA methylation detected in this work might be related to the binding of one or several regulatory factors. We noticed that the undermethylated sites within slr7010 are located next to sll7009 that upon deletion caused an enhanced CRISPR1 expression leading to the classification of S1l7009 as a repressor [34].

We then asked whether the lacking methylation due to mutation of slr0214 would have an impact on the highly efficient DNA interference associated with this system but this was not the case. Moreover, the high transcription of the CRISPR1 repeat-spacer array and its dense and quantitatively high methylation obviously did not interfere with each other. This matches reports for HIP1 to not play a direct role in the regulation of gene expression [4].

However, we detected an approximately $50 \%$ reduced conjugation efficiency in the $\Delta s l r 0214$ mutant compared to the wild type. But, DNA methylation is not restricted to plasmid pSYSA containing the CRISPR system, hence, there is no reason to assume that the lack of M.Ssp6803I-mediated methylation on PSYSA was the only causative for the observed reduced conjugation efficiency. The detected correlation between the genome-wide lacking ${ }^{\mathrm{m} 5}$ CGATCG DNA methylation and the reduced conjugation efficiency cannot be related to DNA integration and recombination because we used a conjugative plasmid that replicates in the cell autonomously and does not require DNA recombination of integration. Moreover, it cannot be related to DNA restriction because M.Ssp6803I is not part of an RM system [7]. Generally, DNA methylation in bacteria can contribute to the transcriptional regulation of genes involved in diverse processes, ranging from biofilm formation, bacteriophage replication, transposition, the timing of chromosome replication and mismatchrepair to conjugation [47]. Therefore it is highly intriguing that instances were described in which DNA methylation affect conjugative transfer, e.g., of a plasmid in Salmonella enterica [48]. In that system, transcription of traJ is increased while transcription of fin $P$ that antagonizes traJ expression, is reduced in a dam mutant. The ratio between the methylation and transcription of these two genes accounts for the level of tra operon expression and the efficiency of conjugation of that plasmid [49, 50]. Lacking DNA methylation leads to measurable changes in gene expression in Synechocystis 6803 as well [38]. Therefore, it is not unlikely that a yet unknown regulatory process that is affected via changed gene expression in mutant $\Delta s l r 0214$ possibly is also affecting plasmid uptake or replication resulting in lowered conjugative efficiency. 


\section{Conclusions}

The majority of bacteria and archaea use CRISPR-Cas systems for antiviral defense. Multiple relationships between DNA methylation, DNA recombination, repair and CRISPR-Cas systems were previously reported. Here, we addressed the possibility of directs links between DNA methylation and the CRISPR-Cas apparatus using the cyanobacterium Synechocystis 6803 as a model. Major DNA methylation sites in this organism are the $\mathrm{GG}^{4 \mathrm{~m}} \mathrm{CC}$ motif recognized by M.Ssp6803II and the HIP1-related motif ${ }^{5 \mathrm{~m}} \mathrm{CG}^{6 \mathrm{~m}} \mathrm{ATCG}$ recognized by M.Ssp6803I and M.Ssp6803III, respectively. We report a remarkable discrepancy in the distribution of GGCC sites along the CRISPR1 cas genes, supporting the hybrid character of subtype I-D CRISPR-Cas systems. The here identified tight and very high ${ }^{5 \mathrm{~m}} \mathrm{C}$ methylation of the CRISPR1 repeat sequences point at some functional relevance. Indeed, while cells lacking the ${ }^{5 \mathrm{~m}} \mathrm{C}$ methylation activity were unaffected in the CRISPR1-mediated interference response, the efficiency of conjugation was reduced to $\sim 50 \%$. Because we used a conjugative plasmid to challenge the CRISPR1 system, the observed difference cannot be related to DNA integration and recombination as might have been involved when integrative transformation of a suicide vector was used as a read-out [39]. Instead, the results point to an unknown role of ${ }^{\mathrm{m} 5}$ CGATCG DNA-methylation marks in conjugation and DNA transformation.

\section{Abbreviations \\ aCas: adaptation Cas; Cas: CRISPR-associated proteins; CRISPR: Clustered regularly interspaced short palindromic repeats; crRNAs: CRISPR RNAs; HIP1: Highly Iterated Palindrome 1; iCas: interference Cas; PAM: Protospacer adjacent motif; pCas: processing Cas}

\section{Authors' contributions \\ IS carried out the molecular genetic and microbiological analyses, JB and KG constructed and provided mutants and constructs, SCL performed the bioinformatics analyses. WRH and $\mathrm{MH}$ designed the study and all authors analyzed data. WRH drafted the manuscript with contributions from all authors. All authors read and approved the final manuscript. \\ Funding \\ This study was funded by the German Research Foundation (Deutsche Forschungsgemeinschaft) via a joint grant to $\mathrm{MH}(\mathrm{HA2002/17-1)}$ and $\mathrm{WRH}$ (HE 2544/10-1), by the German Research Foundation priority program SPP2141 "Much more than Defence: The Multiple Functions and Facets of CRISPR-Cas" (grant HE 2544/14-1) and by the Federal Ministry of Education and Research (BMBF) program de.NBI-Partner grant 031L0106B to WRH. The open access publication fees are covered by the University of Freiburg.}

\section{Availability of data and materials}

Previously generated bisulfite raw data re-analyzed during the current study are available at https://www.ncbi.nlm.nih.gov/biosample/8378604 (BioProject ID: PRJNA430784, BioSample: SAMN08378604, Run: SRX3574087). All other data generated or analyzed during this study are included in this published article.

\section{Ethics approval and consent to participate}

Not applicable.

\section{Consent for publication}

Not applicable.

\section{Competing interests}

The authors declare that they have no competing interests.

\section{Author details}

${ }^{1}$ Faculty of Biology, Genetics an Experimental Bioinformatics, University of Freiburg, Schänzlestr. 1, D-79104 Freiburg, Germany. 'University of Rostock, Institute of Biosciences, Plant Physiology, A.-Einstein-Str. 3, D-18059 Rostock, Germany. ${ }^{3}$ University of Freiburg, Freiburg Institute for Advanced Studies, Albertstr. 19, D-79104 Freiburg, Germany.

Received: 1 February 2019 Accepted: 20 June 2019

Published online: 01 July 2019

\section{References}

1. Robinson NJ, Robinson PJ, Gupta A, Bleasby AJ, Whitton BA, Morby AP. Singular over-representation of an octameric palindrome, HIP1, in DNA from many cyanobacteria. Nucleic Acids Res. 1995;23:729-35.

2. Robinson PJ, Cranenburgh RM, Head IM, Robinson NJ. HIP1 propagates in cyanobacterial DNA via nucleotide substitutions but promotes excision at similar frequencies in Escherichia coli and Synechococcus PCC 7942. Mol Microbiol. 1997;24:181-9.

3. Kaneko T, Tabata S. Complete genome structure of the unicellular cyanobacterium Synechocystis sp. PCC6803. Plant Cell Physiol. 1997;38:1171-6.

4. Xu M, Lawrence JG, Durand D. Selection, periodicity and potential function for highly iterative Palindrome-1 (HIP1) in cyanobacterial genomes. Nucleic Acids Res. 2018:46:2265-78.

5. Elhai J. Highly iterated palindromic sequences (HIPs) and their relationship to DNA methyltransferases. Life Basel Switz. 2015;(5):921-48.

6. Casadesús J. Bacterial DNA methylation and methylomes. Adv Exp Med Biol. 2016:945:35-61.

7. Hagemann M, Gärtner K, Scharnagl M, Bolay P, Lott SC, Fuss J, et al. Identification of the DNA methyltransferases establishing the methylome of the cyanobacterium Synechocystis sp. PCC 6803. DNA Res. 2018;25:343-52.

8. Scharnagl M, Richter S, Hagemann M. The cyanobacterium Synechocystis sp. strain PCC 6803 expresses a DNA methyltransferase specific for the recognition sequence of the restriction endonuclease Pvul. J Bacteriol. 1998; 180:4116-22.

9. Matveyev AV, Young KT, Meng A. Elhai J. DNA methyltransferases of the cyanobacterium Anabaena PCC 7120. Nucleic Acids Res. 2001;29:1491-506.

10. Barrangou R, Fremaux C, Deveau H, Richards M, Boyaval P, Moineau S, et al. CRISPR provides acquired resistance against viruses in prokaryotes. Science. 2007;315:1709-12.

11. Jansen R, van Embden JDA, Gaastra W, Schouls LM. Identification of genes that are associated with DNA repeats in prokaryotes. Mol Microbiol. 2002;43: 1565-75.

12. Mojica FJM, Díez-Villaseñor C, García-Martínez J, Soria E. Intervening sequences of regularly spaced prokaryotic repeats derive from foreign genetic elements. J Mol Evol. 2005:60:174-82.

13. Mojica FJM, Díez-Villaseñor C, García-Martínez J, Almendros C. Short motif sequences determine the targets of the prokaryotic CRISPR defence system. Microbiol Read Engl. 2009;155(Pt 3):733-40.

14. Scholz I, Lange SJ, Hein S, Hess WR, Backofen R. CRISPR-Cas systems in the cyanobacterium Synechocystis sp. PCC6803 exhibit distinct processing pathways involving at least two Cas6 and a Cmr2 protein. PLoS One. 2013;8: e56470.

15. Behler J, Sharma K, Reimann V, Wilde A, Urlaub H, Hess WR. The hostencoded RNase E endonuclease as the crRNA maturation enzyme in a CRISPR-Cas subtype III-Bv system. Nat Microbiol. 2018;3:367-77.

16. Carte J, Wang R, Li H, Terns RM, Terns MP. Cas6 is an endoribonuclease that generates guide RNAs for invader defense in prokaryotes. Genes Dev. 2008; 22:3489-96.

17. Nam KH, Haitjema C, Liu X, Ding F, Wang H, DeLisa MP, et al. Cas5d protein processes pre-crRNA and assembles into a cascade-like interference complex in subtype I-C/Dvulg CRISPR-Cas system. Struct Lond Engl 1993. 2012;20:1574-84.

18. Punetha A, Sivathanu R, Anand B. Active site plasticity enables metaldependent tuning of Cas5 $\mathrm{d}$ nuclease activity in CRISPR-Cas type I-C system. Nucleic Acids Res. 2014:42:3846-56.

19. Jesser R, Behler J, Benda C, Reimann V, Hess WR. Biochemical analysis of the Cas6-1 RNA endonuclease associated with the subtype I-D CRISPR-Cas system in Synechocystis sp. PCC 6803. RNA Biol. 2019;16:481-91. 
20. Reimann V, Alkhnbashi OS, Saunders SJ, Scholz I, Hein S, Backofen R, et al. Structural constraints and enzymatic promiscuity in the Cas6-dependent generation of crRNAs. Nucleic Acids Res. 2017;45:915-25.

21. Hille F, Richter H, Wong SP, Bratovič M, Ressel S, Charpentier E. The biology of CRISPR-Cas: backward and forward. Cell. 2018;172:1239-59.

22. Hille F, Charpentier E. CRISPR-Cas: biology, mechanisms and relevance. Philos Trans R Soc B Biol Sci. 2016;371. https://doi.org/10.1098/rstb.2015.0496.

23. Hochstrasser ML, Doudna JA. Cutting it close: CRISPR-associated endoribonuclease structure and function. Trends Biochem Sci. 2015;40:58-66.

24. Kieper SN, Almendros C, Behler J, McKenzie RE, Nóbrega FL, Haagsma AC, et al. Cas4 facilitates PAM-compatible spacer selection during CRISPR adaptation. Cell Rep. 2018;22:3377-38.

25. Makarova KS, Gao L, Zhang F, Koonin EV. Unexpected connections between type VI-B CRISPR-Cas systems, bacterial natural competence, ubiquitin signaling network and DNA modification through a distinct family of membrane proteins. FEMS Microbiol Lett. 2019;366. https://doi.org/10.1093/ femsle/fnz088.

26. Rippka R, Deruelles J, Waterbury JB, Herdman M, Stanier RY. Generic assignments, strain histories and properties of pure cultures of cyanobacteria. Microbiology 1979;111:1-61.

27. Zinchenko W, Piven IV, Melnik VA, Shestakov SV. Vectors for the complementation analysis of cyanobacterial mutants. Russ J Genet. 1999;35: 228-32.

28. Bolger AM, Lohse M, Usadel B. Trimmomatic: a flexible trimmer for Illumina sequence data. Bioinformatics. 2014;30:2114-20.

29. Krueger F, Andrews SR. Bismark: a flexible aligner and methylation caller for bisulfite-Seq applications. Bioinforma Oxf Engl. 2011;27:1571-2.

30. Langmead B, Salzberg SL. Fast gapped-read alignment with bowtie 2. Nat Methods. 2012;9:357-9.

31. Vilkaitis G, Klimasauskas S. Bisulfite sequencing protocol displays both 5methylcytosine and N4-methylcytosine. Anal Biochem. 1999;271:116-9.

32. Kopfmann S, Hess WR. Toxin-antitoxin systems on the large defense plasmid pSYSA of Synechocystis sp. PCC 6803. J Biol Chem. 2013;288:7399-409.

33. Garrett RA, Vestergaard G, Shah SA. Archaeal CRISPR-based immune systems: exchangeable functional modules. Trends Microbiol. 2011;19:549-56.

34. Hein S, Scholz I, Voß B, Hess WR. Adaptation and modification of three CRISPR loci in two closely related cyanobacteria. RNA Biol. 2013;10:852-64.

35. Makarova KS, Anantharaman V, Grishin NV, Koonin EV, Aravind L. CARF and WYL domains: ligand-binding regulators of prokaryotic defense systems. Front Genet. 2014;5:102.

36. Sternberg SH, Haurwitz RE, Doudna JA. Mechanism of substrate selection by a highly specific CRISPR endoribonuclease. RNA. 2012;18:661-72.

37. Zerulla K, Ludt K, Soppa J. The ploidy level of Synechocystis sp. PCC 6803 is highly variable and is influenced by growth phase and by chemical and physical external parameters. Microbiol. 2016;162:730-9.

38. Gärtner K, Klähn S, Watanabe S, Mikkat S, Scholz I, Hess WR, et al. Cytosine N4-methylation via M.Ssp6803II is involved in the regulation of transcription, fine-tuning of DNA replication and DNA repair in the cyanobacterium Synechocystis sp. PCC 6803. Front Microbiol. 2019;10:1233.

39. Wang B, Yu J, Zhang W, Meldrum DR. Premethylation of foreign DNA improves integrative transformation efficiency in Synechocystis sp. strain PCC 6803. Appl Environ Microbiol. 2015;81:8500-6.

40. Levy A, Goren MG, Yosef I, Auster O, Manor M, Amitai G, et al. CRISPR adaptation biases explain preference for acquisition of foreign DNA. Nature. 2015;520:505-10.

41. Ivančić-Baće I, Cass SD, Wearne SJ, Bolt EL. Different genome stability proteins underpin primed and naïve adaptation in E. coli CRISPR-Cas immunity. Nucleic Acids Res. 2015;43:10821-30.

42. Radovcic M, Killelea T, Savitskaya E, Wettstein L, Bolt EL. Ivancic-Bace I. CRISPR-Cas adaptation in Escherichia coli requires RecBCD helicase but not nuclease activity, is independent of homologous recombination, and is antagonized by $5^{\prime}$ ssDNA exonucleases. Nucleic Acids Res. 2018;46:10173-83.

43. Babu M, Beloglazova N, Flick R, Graham C, Skarina T, Nocek B, et al. A dual function of the CRISPR-Cas system in bacterial antivirus immunity and DNA repair. Mol Microbiol. 2011;79:484-502.

44. Goldfarb T, Sberro H, Weinstock E, Cohen O, Doron S, Charpak-Amikam Y, et al. BREX is a novel phage resistance system widespread in microbial genomes. EMBO J. 2015;34:169-83.

45. Gordeeva J, Morozova N, Sierro N, Isaev A, Sinkunas T, Tsvetkova K, et al. BREX system of Escherichia coli distinguishes self from non-self by methylation of a specific DNA site. Nucleic Acids Res. 2019;47:253-65.
46. Makarova KS, Haft DH, Barrangou R, Brouns SJJ, Charpentier E, Horvath P, et al. Evolution and classification of the CRISPR-Cas systems. Nat Rev Microbiol. 2011;9:467-77.

47. Marinus MG, Løbner-Olesen A. DNA methylation. EcoSal Plus. 2014;6. https://doi.org/10.1128/ecosalplus.ESP-0003-2013.

48. Camacho EM, Casadesús J. Conjugal transfer of the virulence plasmid of Salmonella enterica is regulated by the leucine-responsive regulatory protein and DNA adenine methylation. Mol Microbiol. 2002;44:1589-98.

49. Camacho EM, Serna A, Madrid C, Marqués S, Fernández R, de la Cruz F, et al. Regulation of finP transcription by DNA adenine methylation in the virulence plasmid of Salmonella enterica. J Bacteriol. 2005;187:5691-9.

50. Camacho EM, Casadesús J. Regulation of traJ transcription in the Salmonella virulence plasmid by strand-specific DNA adenine hemimethylation. Mol Microbiol. 2005;57:1700-18.

51. Kaneko T, Nakamura Y, Sasamoto S, Watanabe A, Kohara M, Matsumoto M, et al. Structural analysis of four large plasmids harboring in a unicellular cyanobacterium, Synechocystis sp. PCC 6803. DNA Res. 2003;10:221-8.

\section{Publisher's Note}

Springer Nature remains neutral with regard to jurisdictional claims in published maps and institutional affiliations.

\section{Ready to submit your research? Choose BMC and benefit from:}

- fast, convenient online submission

- thorough peer review by experienced researchers in your field

- rapid publication on acceptance

- support for research data, including large and complex data types

- gold Open Access which fosters wider collaboration and increased citations

- maximum visibility for your research: over $100 \mathrm{M}$ website views per year

At BMC, research is always in progress.

Learn more biomedcentral.com/submissions 